\title{
Actitudes hacia el amor y apego
}

\author{
María Elena Brenlla * \\ Analía Brizzio ** \\ Alejandra Carreras ***
}

\section{Resumen}

A partir de las concepciones de Lee que propuso una tipología del amor de seis categorías Eros (amor pasional), Ludus (amor lúdico), Storge (amor - amistad), Manía (amor posesivo y dependiente), Pragma (amor lógico y práctico) y Ágape (amor desinteresado y altruista), Hendrick y Hendrick diseñaron la escala de "Actitudes sobre el Amor". Esta evalúa la tipología descripta e informa, mediante sendos ítems, de la frecuencia y las opiniones de las relaciones amorosas y del estilo de apego predominante.

Los objetivos de este estudio consistieron en analizar las propiedades psicométricas del instrumento y las asociaciones entre las actitudes sobre el amor y el autoinforme sobre tipo y frecuencia de relaciones amorosas, por un lado y con el tipo de apego, por el otro.

Los resultados del estudio ( $\mathrm{N}=280$ ) indican una consistencia interna aceptable para el puntaje total (alfa $=0,73) \mathrm{y}$, el análisis factorial realizado, señala que los seis factores considerados explican aproximadamente el $41 \%$ del total de la varianza.

También, se encontró una asociación significativa entre las puntuaciones de Eros y la autopercepción de un estilo seguro en las relaciones amorosas.

Palabras clave: Tipologías sobre el amor - Actitudes sobre el amor - Tipos de apego $\cdot$ Evaluación $\cdot$ Propiedades psicométricas

* Psicóloga. Docente. Facultad de Psicología. Universidad de Buenos Aires

Profesora. Universidad Católica Argentina. E-mail: bren@psi.uba.ar

** Docente . Facultad de Psicología. Universidad de Buenos Aires. Becaria del CONICET.

*** Docente. Facultad de Psicología. Universidad de Buenos Aires. 


\section{Abstract}

\section{Love attitudes and attachment}

Love styles described by Lee are Eros (passionate love), Ludus (game-playing love), Storge (friendship love), Mania (possessive, dependent love), Pragma (logical, "shopping list" love) and Agape (all-giving, selfless love). Based on those types, Hendrick and Hendrick developed a 42-ítem rating questionnaire with 7 items measuring each love style (Love Attitudes Scale). Beside, inform about frequency in love relationships and attachment style.

The purpose of this study was analyze the reliability and factor structure of the Love Attitudes Scale and to investigate the association between love attitudes and the attachment style. The results ( $\mathrm{N}=280$ participants) indicate adequate internal consistency (alfa $=0,73$ ). The items were intercorrelated and factored. The best solution extracted six factors using varimax rotation and all six factors accounted $41 \%$ of the total variance. Secure attachment was related positively to eros.

Key words: Love styles ' Love attitudes ' Attachment ' Assessment · Psychometric properties 


\section{Actitudes hacia el amor y apego}

El amor, en términos generales, es definido como una fuerte inclinación emocional hacia otra persona y, en un sentido más amplio, hacia un animal o hacia grupos de personas u objetos. Por lo general, se diferencia entre amor erótico hacia el compañero sexual y amor no sexual, como por ejemplo el de los padres hacia sus hijos (y viceversa). Este segundo caso incluye el concepto de 'amor platónico' que puede existir entre dos partners sexuales potenciales. El amor en sus diferentes acepciones es objeto de estudios religiosos, filosóficos y psicológicos.

En la antigüedad griega y en la filosofía hindú, el amor representa el principio del cosmos. El amor a sí mismo es, según Aristóteles, requisito indispensable para poder amar a otra persona, aunque no debe confundirse con el egoísmo. En el cristianismo, Dios ama a los hombres compadeciéndose de ellos; sin embargo, el amor de los cristianos hacia Dios está caracterizado por el respeto y la adoración. La disposición de ayudar a los demás se denomina amor al prójimo.

En psicología, la mayor parte de los estudios realizados se centra en el amor sexual y en el impulso sexual subyacente. La formación individual de la libido es la base de la teoría del psicoanálisis. Actualmente, la investigación psicológica de las relaciones interpersonales asnaliza el tema del amor, principalmente en el proceso de formación y finalización de las relaciones de una pareja de larga duración.

En las últimas décadas el amor se ha convertido en un tema relevante de estudio para la Psicología Social. Se han propuesto numerosas teorías sobre el tema, variando desde un concepto global hasta teorías que analizan el constructo de un modo multidimensional, promoviendo campos de conocimientos más vastos.

\section{Definición del amor}

Son muchas las aproximaciones que se realizaron al estudio del amor sin poder converger en un criterio único o en una única definición.

La Real Academia Española propone varias acepciones para la palabra amor. Entre ellas se destacan dos principales: 1. sentimiento que mueve a desear que la realidad amada, otra persona, un grupo humano o alguna cosa, alcance lo que se juzga su bien, a procurar que ese deseo se cumpla y a gozar como bien propio el hecho de saberlo cumplido. 2. Atracción sexual.

En esta misma línea, muchos científicos distinguen entre dos formas del amor: "amor pasional" y "amor compañero". El amor pasional se define como un estado caracterizado por el anhelo intenso de unión con el otro, mientras que el 
amor compañero se define desde una perspectiva evolucionista centrado en la reproducción. (Hatfield y Rapson, 1999). Otros investigadores propusieron tipologías más elaboradas de la complejidad del concepto amor (Sternberg,1988 y Hendrick, 1989).

Otros abordajes han distinguido entre amar y gustar (Rubin, 1970). Claramente esta distinción es viable pues separa la literatura desarrollada tanto en amistad como en atracción romántica ya que ambas literaturas tienen muy poco en común (Zubieta, 2001). También puede hacerse una distinción entre amor y compromiso aunque algunas teorías (Sternberg, 1986) ven el compromiso como parte del amor.

Hendrick y Hendrick (1992) no proveen una definición formal del amor sino que proponen de manera general razones para la existencia del amor a través de dos amplios abordajes teóricos. Una aportación biológica que considera al amor como algo que fluye directamente de nuestros genes, como parte de nuestra herencia evolutiva y, en contraste, una aportación sociológica que sostiene que el amor simplemente fluye de ciertos patrones de relaciones sociales y que involucra definiciones del sí mismo (self) y del rol de uno mismo respecto de los otros.

El amor también ha sido definido como una faceta de la personalidad, como una conducta aprendida y hasta como una parte de nuestra herencia evolutiva (Mellen, 1981).

\section{Evaluación del amor}

Si bien se han realizado numerosas teorizaciones en relación con este tema, no se han desarrollado, en simultaneidad, instrumentos válidos para su medición.

Una de las teorías del amor que posibilitó medir el concepto, fue la propuesta por Lee $(1973 ; 1976)$, quien desarrolló una clasificación de las diferentes aproximaciones al amor. Luego de un procedimiento extenso de entrevistas y de técnicas complejas de análisis de datos, Lee propuso una tipología de los estilos del amor que conforma un círculo cerrado. Este autor identificó tres tipos primarios de estilo de amor: Eros (amor romántico y pasional), Ludus (amor lúdico), Storge o Amistad (amor - amistad); y tres estilos secundarios: Manía (amor posesivo y dependiente), Pragma (amor lógico y práctico) y Ágape (amor altruista). Estos estilos secundarios fueron concebidos como combinados formados por pares de estilos primarios. Haciendo una analogía con los compuestos químicos, los estilos secundarios son transformaciones cua- 
litativas de los elementos primarios básicos. El tipo Manía es una combinación de Eros y Ludus aunque se diferencia cualitativamente de los dos primarios. Del mismo modo, Pragma resulta de la conjunción de Storge y Ludus aunque con propiedades muy diferentes. Lo mismo sucede con Ágape, un compuesto de Eros y Storge. Una implicación importante de esta analogía con los compuestos químicos es que los seis estilos del amor se correlacionan de manera lógica pero cada estilo mantiene propiedades cualitativas independientes de los otros. En suma los estilos de amor son formas igualmente válidas de amar, es decir, no hay un solo prototipo de amor sino diferentes modalidades (Hendrick y Hendrick, 1986).

A partir de la tipología propuesta por Lee han sido varios los intentos de cuantificar los diferentes modos del amor. Sin embargo, es la Escala de Actitudes sobre el Amor de Hendrick y Hendrick (1992) la que evalúa esta tipología desde un abordaje individual de las relaciones amorosas. En realidad, se tratan los seis tipos del amor como diferentes variables más que como una tipología, es decir, a partir de una escala que evalúa cada una de las formas de amar, los individuos pueden obtener un perfil similar a los seis tipos diferentes.

Los ítems de la Escala reflejan un seguimiento fiel de la concepción teórica de cada estilo de amor.

Eros o el amor romántico y la pasión presentan una relación en la cual la apariencia física desempeña un rol importante como así también el componente sexual y el deseo de una relación intensa. Una tendencia al estilo Eros marca la valoración del amor pero no el estar obsesionado por él ni la presión a la pareja por la intensidad, sino más bien se permite que las cosas se desarrollen mutuamente, de forma espontánea. La característica de este tipo es la alta confianza y la alta autoestima. En $L u d u s$ o el amor lúdico, amor y sexualidad son vistos como un juego para el disfrute mutuo y sin una intención seria particular. Esta tendencia amorosa puede concretarse con más de una pareja a la vez, puede disfrutar de diferentes personas, actividades y placeres. Este estilo de amar no se relaciona directamente con un tipo físico preferido y ve al sexo más como "una forma de divertirse" que como una profunda implicación. Aún cuando mucha gente considera este amor como moralmente negativo, Ludus no intenta herir a otras personas, generalmente pone muy claras las reglas del juego antes de comenzar la relación. Storge (término que proviene de una palabra griega que denota afecto parental) hace referencia al amoramistad, a un compromiso durable que se desarrolla lenta y prudentemente. Es más un amor por evolución que por revolución. El punto fuerte de este amor es el compañerismo y la relación de confianza que se establece con la pareja, quien es similar en términos de valores y actitudes. Esta similitud es mucho más importante para Storge que la apariencia física o la satisfacción sexual porque la orientación de este amor es la de buscar un compromiso a largo 
plazo. Pragma alude a un amor práctico, tomando en consideración la edad, el grado de instrucción, el estatus social, la religión o la facultad de ser una buena madre o un buen padre. Manía es el amor posesivo, la tendencia a experimentar celos, los estados emocionales intensos, la necesidad constante de asegurar el amor del otro. La tendencia al estilo maníaco fuerza a la pareja al compromiso sin poder esperar que este evolucione naturalmente, lo que lleva en ocasiones, a que la relación se termine, confirmando los temores del amante maníaco. Ágape es el estilo más raro de la tipología de Lee, es un amor altruista, que se preocupa por el bienestar del otro y no exige nada a cambio, es un amor más bien idealista en el que la sexualidad y la sensualidad no son relevantes (Zubieta, 2001).

\section{Estudios previos}

Un estudio realizado por Hendrick y Hendrick (1986) en la Universidad de Miami sobre una muestra de 466 estudiantes universitarios, evidenció la existencia de diferencias significativas en los estilos de amor entre hombres y mujeres. En general los hombres poseen un estilo de amar más lúdico que las mujeres. Por otro lado, las mujeres presentan tendencias significativas hacia los estilos Pragma, Storge y Manía. Asimismo, hombres y mujeres no se diferencian en los estilos Eros y Agape. Los patrones de estos resultados son muy similares a los encontrados en estudios previos (Hendrick et al.,1984).

Con respecto a la edad de los sujetos evaluados no se encontraron diferencias significativas respecto de los estilos de amor (Hendrick y Hendrick, 1986).

En un estudio realizado en España con una muestra compuesta por 144 estudiantes universitarios de la Comunidad Autónoma Vasca, se obtuvieron diferencias significativas por sexo en solo dos estilos de amor: Amistad y Ágape, en ambos casos eran los hombres lo que presentaban mayores tendencias hacia estas modalidades (Zubieta, 2001).

\section{Actitudes hacia el Amor y Estilos de Apego}

Los estilos del amor pueden relacionarse con los diferentes tipos de Apego. La teoría sobre el APEGO se focaliza en el estudio de los procesos a través de los cuales niños e infantes desarrollan sentimientos de confianza en la protección paterna y/o adulta. La teoría sobre el Apego, formulada inicialmente por el psiquiatra británico Bowlby $(1969 ; 1982)$ y continuada luego por la investigadora canadiense Ainsworth (1989), intentan explicar los efectos de los vínculos tempranos de protección en el desarrollo psicológico del neonato y el 
infante, así como los efectos que produce el no contar con tales vínculos (Brenlla, Brizzio y Carreras; 2001). La combinación de dos dimensiones propuestas por Ainsworth -Ansiedad y Evitación- dan como resultado cuatro patrones diferentes de apego: seguro, ansioso, evitativo y temeroso. Estudios realizados en los fines de los ' 80 y principios de los '90 revelan cómo cada estilo de apego en el adulto correlaciona con importantes constructos relacionales como el compromiso, la satisfacción, la confianza y la estabilidad a largo plazo en las relaciones románticas (Simpson, Rholes; año). Shaver \& Hazan (1988) propusieron que las teorías sobre el amor podían ser integradas en la perspectiva de la teoría del apego y arguyeron que la tipología de Lee podía ser reducida a tres estilos de apego. El apego seguro debía corresponder a una combinación entre Eros y Agape; el evitativo con Ludus y el ambivalente a Manía. No incluyeron Pragma ni Storge por no considerarlos como formas de amor romántico. Levy \& Davis (1988) obtuvieron resultados en los que el apego seguro fue asociado positivamente con Eros y Agape y negativamente con Ludus; el tipo evitativo fue asociado positivamente a Ludus y negativamente a Eros y el ambivalente se encontró relacionado con Manía. No obstante, esas relaciones fueron modestas en términos cuantitativos. Por su parte, Sternberg (1986) encontró que su modelo del amor de tres componentes (intimidad pasión - compromiso), se asociaba positivamente con el tipo de apego seguro y negativamente con el tipo ambivalente. Parece probable, entonces que exista una asociación positiva entre el tipo de apego seguro y una actitud hacia el amor en términos de pasión y romanticismo (Eros) así como entre ambivalente y un estilo posesivo y dependiente (Manía).

En este estudio, nos propusimos como objetivos principales estudiar si a semejanza de los que ocurre en otros países, los hombres muestran una preferencia hacia Ludus y las mujeres hacia Eros; conocer el tipo de actitud hacia el amor y el estilo de apego predominantes en adultos jóvenes y analizar las propiedades psicométricas de la escala "Actitudes sobre el amor" de Hendrick y Hendrick (1986) en sujetos adultos, en particular la consistencia interna y la estructura factorial de sus ítems.

\section{Método}

\section{Participantes}

Participaron del estudio 280 adultos jóvenes (edad, $\mathrm{M}=27,4 ; \mathrm{DE}=5,66$ ), 140 varones $(50 \%)$ y 140 mujeres $(50 \%)$, residentes en la Ciudad de Buenos Aires y Conurbano bonaerense. El estado civil de los participantes se categorizó como soltero (70\%), casado (23\%), en pareja (2\%), divorciado (3\%), separa- 
do $(2 \%)$ y el nivel educativo en función de primario completo - secundario incompleto (8\%); secundario completo - terciario incompleto $(69 \%)$ y terciario completo $(23 \%)$. La categoría "terciario" incluye estudios de tercera enseñanza y universitarios.

\section{Instrumentos}

Escala de Actitudes sobre el amor (ASA)

Se trata de una escala de 42 ítems que se contesta en un formato tipo likert. Esos ítems están agrupados según la tipología propuesta por Lee en Eros, Ludus, Amistad; Pragma; Manía y Agape. Se ha analizado la validez y la fiabilidad del instrumento en población estadounidense (Hendrick y Hendrick, 1986) y española (Zubieta, 2001).

La versión administrada incluye además seis preguntas acerca de frecuencias y preferencias en las relaciones románticas y tres ítems que indagan tres tipos de apego (seguro, evitativo, ambivalente).

\section{Procedimiento}

Los datos fueron recolectados durante los años 2001 - 2002. Se instruyó a alumnos de la Facultad de Psicología de la Universidad de Buenos Aires para que administrasen las técnicas descriptas como parte de una práctica sobre pruebas autoadministrables en la materia de la cual una de las autores es profesora titular (dra. M.M. Casullo). Luego se examinaron los protocolos y se desecharon aquellos en los que se hubiese omitido algún dato sociodemográfico relevante a los fines del estudio (sexo, edad) o los que por otras razones resultasen inapropiados (incompletud, etc.)

Se utilizaron planillas de cálculo para permitir una tabulación más rápida y precisa. Las puntuaciones obtenidas fueron analizadas con el paquete estadístico SPSS- 10 .

\section{Resultados}

Al iniciar este estudio nos planteamos como objetivos estudiar si, en concordancia con los resultados obtenidos en otros países, los hombres mostraban una actitud hacia el amor cuyo eje central fuera la diversión y una explícita falta de compromiso en las relaciones amorosas (LUDUS) y las mujeres, una preferencia hacia el amor romántico y pasional (EROS); relacionar el tipo de actitud hacia el amor y el estilo de apego en adultos jóvenes y analizar las propie-

dades psicométricas de la escala "Actitudes sobre el amor" de Hendrick y 
Hendrick en sujetos adultos, en particular, la consistencia interna y la estructura factorial de sus ítems.

\section{1) Análisis descriptivos}

Comenzaremos por señalar algunas características de los sujetos inferidas a partir sus respuestas a los ítems 43 a 47 de ASA. En la tabla 1 a 5 se detallan las frecuencias y porcentajes según sexo para estos ítems.

Como puede verse allí, tanto hombres como mujeres señalaron en mayor proporción que estuvieron enamorados una o dos veces (aprox. 70\% en ambos sexos) y que están enamorados actualmente (aprox. 70\% para varones y mujeres) En cambio, acerca de la cantidad de personas con las que habían mantenido relaciones sexuales, los hombres respondieron, en su mayoría, una frecuencia mayor que cinco (aprox. 71\%) y las mujeres, indicaron en mayor proporción la frecuencia de cuatro o menos (aprox. 80\%). Por otra parte, tanto varones cuanto mujeres coincidieron en la frecuencia de sus respuestas a las preguntas: Si una persona tuviese todas las características que usted considera deseables, ¿Viviría con ella aunque no estuviese enamorado? y Si el amor se termina ¿es preferible para ambos separarse y comenzar una nueva vida?, indicando para la primera mayoritariamente "No" (varones $80 \%$; mujeres $85 \%$ ) y "Sí" para la segunda (varones 94\%; mujeres 91\%).

Tabla 1. Análisis de los items de la escala de actitudes hacia el amor (item 43)

\begin{tabular}{lrrrr}
\hline & \multicolumn{3}{c}{ Cuántas veces ha estado enamorado } \\
\hline & \multicolumn{2}{c}{ Varones } & \multicolumn{2}{c}{ Mujeres } \\
\hline & f & $\%$ & f & $\%$ \\
Nunca & 3 & 2,1 & 3 & 2,1 \\
1 vez & 55 & 39,3 & 51 & 36,4 \\
2 veces & 45 & 32,1 & 47 & 33,6 \\
3 veces & 25 & 17,9 & 21 & 15 \\
4 o más & 12 & 8,6 & 18 & 12,9 \\
\hline Total & 140 & 100 & 140 & 100 \\
\hline
\end{tabular}


Tabla 2. Análisis de los items de la escala de actitudes hacia el amor (item 44)

\begin{tabular}{lrrrrr}
\hline \multicolumn{4}{c}{$\begin{array}{c}\text { ¿A lo largo de su vida, ¿con cuántas personas, } \\
\text { aproximadamente, ha tenido relaciones sexuales? }\end{array}$} \\
\hline \multicolumn{4}{c}{ Varones } & \multicolumn{3}{c}{ Mujeres } \\
\hline & f & $\%$ & f & $\%$ \\
Con nadie & 1 & 0,7 & 4 & 2,9 \\
1 & 15 & 10,7 & 39 & 27,9 \\
2 a 4 & 24 & 17,1 & 51 & 36,4 \\
5 a 10 & 52 & 37,1 & 27 & 19,3 \\
11 o más & 48 & 34,3 & 19 & 13,6 \\
\hline Total & 140 & 100 & 140 & 100 \\
\hline
\end{tabular}

Tabla 3. Análisis de los items de la escala de actitudes hacia el amor (item 45)

\begin{tabular}{lcc}
\hline & \multicolumn{2}{c}{ ¿Está enamorado actualmente? } \\
\hline & Varones & Mujeres \\
\hline \multirow{2}{*}{ si } & $\%$ & $\%$ \\
no & 67,1 & 73,6 \\
N/C & 32,1 & 25 \\
\hline Total & 0,7 & 1,4 \\
\hline
\end{tabular}

Tabla 4. Análisis de los items de la escala de actitudes hacia el amor (item 46)

\begin{tabular}{ccc}
\hline & \multicolumn{2}{c}{$\begin{array}{c}\text { Si una persona tuviese todas las características } \\
\text { que usted considera deseables, ¿Viviría con ella } \\
\text { aunque no estuviese enamorado? }\end{array}$} \\
\hline & Varones & Mujeres \\
\hline si & $\%$ & 15,7 \\
no & 20,0 & 84,3 \\
\hline Total & 80,0 & 100 \\
\hline
\end{tabular}


Tabla 5. Análisis de los items de la escala de actitudes hacia el amor (item 47)

\begin{tabular}{lcc}
\hline \multicolumn{3}{c}{$\begin{array}{c}\text { Si el amor se termina ¿es preferible para ambos } \\
\text { separarse y comenzar una nueva vida? }\end{array}$} \\
\hline & Varones & Mujeres \\
\hline & $\%$ & $\%$ \\
si & 94,3 & 91,4 \\
no & 5,7 & 8,6 \\
\hline Total & 100 & 100 \\
\hline
\end{tabular}

\section{2) Comparaciones según sexo}

En la tabla 6 se brindan los datos descriptivos de ASA (todos los casos) y en la tabla 7 diferenciados según sexo. Como puede observarse, las medidas de la forma de la distribución, indican que las puntuaciones de las escalas de EROS y LUDUS tienden a concentrarse en torno a la media de modo mayor a como sería esperable en una distribución normal mientras que el análisis de la asimetría indica, respecto de EROS y $A G A P E$, que las desviaciones a la media son mayores para los valores superiores que para los inferiores. Por ende, debe inferirse que la distribución de las puntuaciones no es normal en el caso de EROS, LUDUS Y AGAPE. Lo mismo ocurre si se analiza el índice de Kolmogorov - Smirnov para esas dimensiones. Por lo tanto, es preferible utilizar medidas no paramétricas para analizar las comparaciones entre medias en estos casos. Entonces, para apreciar si hombres y mujeres diferían en sus puntuaciones en esas escalas de ASA, tal como ocurre en varios estudios precedentes, se utilizó la prueba de MannWhitney. Los resultados indican que no hay diferencias significativas según sexo a las escalas de ASA. Hubo una sola subescala que presentó una diferencia importante aunque no significativa: $A G A P E$, con puntuaciones promedio mayores para mujeres que para varones. En las restantes dimensiones, PRAGMA, MANIA Y AMISTAD, la distribución es normal por lo que se calculó el estadístico " $\mathrm{t}$ ". Tampoco en este caso, se observaron diferencias significativas por sexo. 
Tabla 6: Estadísticos descriptivos de la escala ASA para varones y mujeres combinados

\begin{tabular}{lcc}
\hline & Media & $\begin{array}{c}\text { Desvío } \\
\text { Estándar }\end{array}$ \\
\hline EROS & 14,30 & 4,2 \\
LUDUS & 24,08 & 4,4 \\
AMISTAD & 22,72 & 5,2 \\
PRAGMA & 23,16 & 5,9 \\
MANIA & 21,21 & 5,2 \\
AGAPE & 17,18 & 5,0 \\
\hline $\mathrm{N}=280$ & & \\
\hline
\end{tabular}

Tabla 7. Estadísticos descriptivos ASA según sexo

\begin{tabular}{lcccc}
\hline & Sexo & $\mathrm{N}$ & Media & $\begin{array}{c}\text { Desvío } \\
\text { Estándar }\end{array}$ \\
\hline EROS & masculino & 140 & 14,6 & 4,11 \\
& femenino & 140 & 14,14 & 4,43 \\
LUDUS & masculino & 140 & 23,71 & 4,45 \\
& femenino & 140 & 24,44 & 4,52 \\
PMISTAD & masculino & 140 & 22,21 & 5,21 \\
& femenino & 140 & 23,23 & 5,26 \\
MANIA & masculino & 140 & 23,71 & 6,02 \\
& femenino & 140 & 22,62 & 5,81 \\
AGAPE & masculino & 140 & 21,51 & 5,19 \\
& femenino & 140 & 20,91 & 5,25 \\
& masculino & 140 & 16,59 & 4,97 \\
& femenino & 140 & 17,77 & 5,10 \\
\hline
\end{tabular}


3) Medidas de asociación entre "Actitudes sobre el amor" y "tipos de apego"

El ítem 48 se compone de tres frases entre las que el sujeto tiene que elegir cuál se aplica más a su persona y que reflejan un tipo de apego seguro, ambivalente o evitativo. Tal como lo ilustra la tabla 8, existe un predominio del tipo de apego seguro en varones y mujeres.

Para analizar la relación entre tipos de apego y actitudes hacia el amor, se calculó el coeficiente Eta dado que se trataba de ver si existía asociación entre una variable nominal (tipos de apego) y otra medida cuantitativamente (actitudes sobre el amor). Los resultados indican una asociación relevante entre el tipo de apego "seguro" y el tipo EROS (0,288; signif. aprox. 0,003). Esta asociación significa que un determinado puntaje en la escala Eros, depende, moderadamente, de cuál haya sido la categoría señalada como tipo de apego predominante, en este caso seguro. No se registraron asociaciones significativas entre los demás tipos de apego y escalas de actitudes hacia el amor.

Tabla 8 Frecuencias y porcentajes de tipos de apego

\begin{tabular}{lrrrr}
\hline \multicolumn{1}{c}{ Item 48 } & \multicolumn{2}{c}{ Varones } & \multicolumn{2}{c}{ Mujeres } \\
\hline & \multicolumn{1}{c}{$\mathrm{f}$} & \multicolumn{1}{c}{$\%$} & \multicolumn{1}{c}{$\mathrm{f}$} & $\%$ \\
Ninguna & 1 & 0,7 & 3 & 2,1 \\
48 A (seguro) & 72 & 51,4 & 71 & 50,7 \\
48 B (evitativo) & 37 & 26,4 & 37 & 26,4 \\
48 C (ambivalente) & 21 & 15,0 & 16 & 11,4 \\
N/C & 9 & 6,4 & 13 & 9,3 \\
\hline Total 14 & $\mathbf{1 0 0}$ & $\mathbf{1 0 0}$ & $\mathbf{1 4 0}$ & $\mathbf{1 0 0}$ \\
\hline
\end{tabular}

48 A: Me resulta fácil tener relaciones íntimas. Necesito estar unido a otro/a 48 B: No me siento muy a gusto con la idea de intimar mucho con las personas. Mis parejas hubieran querido que yo hubiera estado más cercano a ellas, pero me hace sentir incómodo

$48 \mathrm{C}$ : Encuentro que los otros dudan antes de intimar conmigo como yo quisiera que lo hicieran. A veces tengo la impresión de que mi pareja no me ama ( o no me ha amado) lo suficiente 
4) Propiedades psicométricas de "Actitudes sobre el Amor" (ASA)

\section{Fiabilidad}

Con la finalidad de estimar la consistencia interna se empleó el procedimiento alfa de Cronbach, que proporciona una estimación del grado en que covarían los ítems de un test o escala (Muñiz, 2001). Los resultados para el puntaje total se consideran aceptables (alfa $=0,73$ ) al igual que para cada uno de los tipos (alfa entre 0,64 y 0,72 ) salvo en el caso de LUDUS, donde se encontró un valor francamente bajo $(0,40)$.

\section{Estructura factorial de los items de ASA}

Los 42 ítems de ASA fueron sometidos a análisis de componentes principales con extracción de seis factores en función de la tipología empleada y, luego rotados usando la rotación Varimax ya que se supone independencia de los factores. Previamente se comprobó que la matriz de correlaciones era la adecuada para este análisis (Test de esfericidad de Bartlett $=3054,32 \mathrm{p}<0,001$; índice de adecuación muestral $\mathrm{KMO}=0,70)$. Luego de la rotación empleada, el porcentaje de la varianza explicada por cada factor es como sigue: Eros $(6,2)$, Ludus $(3,8)$; Amistad (5,0); Pragma $(9,6)$; Manía $(4,7)$ y Agape $(11,1)$. Por lo tanto, los seis factores explican aproximadamente el $41 \%$ del total de la varianza. Agape fue el primer factor extraído y, por definición, el que explica más varianza. Esto también se observó en el estudio de Hendrick y Hendrick, (1986). Después, aparecen Pragma, Eros, Amistad, Manía y Ludus. La tabla I ilustra estos resultados. De los 42 ítems que forman el instrumento, la mayor parte mostraron cargas factoriales cercanas o mayores de 0,35 , oscilando los valores entre 0,34 y 0,77 . Sólo cinco mostraron cargas factoriales bajas: los ítems 9 y 10 (Ludus) y 15, 16 y 17 (Amistad). No obstante ello, se decidió mantener estos ítems en el instrumento, tal como se realizó en estudios previos (Hendrick y Hendrick, 1986). Como puede notarse en la Tabla $\mathrm{V}$ existe escasa superposición de los ítems en función de los factores considerados.

Ahora, si se analiza la posición en la que aparecieron los ítems luego de la rotación y la correspondencia con la tipología de Lee implícita en las subescalas de ASA, puede notarse que existe una congruencia importante entre la solución factorial hallada y los ítems supuestos en la prueba para cada actitud sobre el amor.

El primer factor contiene ocho ítems de los cuales cinco se corresponden con ítems de la subescala Agape (AG), dos con Eros (ER) y uno con Manía (MA). Recuérdese que AG se relaciona con actitudes sobre el amor caracterizadas por el altruismo, la devoción y el idealismo. Si se analiza el contenido de los ítems correspondientes a MA y a ER puede inferirse que guardan relación con los de 
AG, ya que su contenido alude a estos aspectos.

El segundo factor está compuesto por nueve ítems de los cuales siete son la totalidad de los ítems de Pragma (PR) y los dos restantes se corresponden con sendos de Ludus (LU). En este factor el predominio de contenido se relaciona con aspectos pragmáticos y realistas respecto de las actitudes hacia el amor.

El tercer factor contiene siete ítems. De éstos, cinco corresponden a Eros (ER) y reflejan actitudes sobre el amor donde la pasión, la apariencia física, el componente sexual y la confianza en sí son los aspectos centrales. Los dos restantes corresponden a AG y a Amistad (AM). En este caso el contenido de los minoritarios aluden a nociones de compañerismo que quizá puedan considerarse afines a las de ER.

El cuarto factor se compone de seis ítems que coinciden con seis de los siete que forman la subescala AM. El contenido de ellos enfatiza el compañerismo, la similitud de valores y actitudes y el compromiso a largo plazo.

El quinto factor consiste en seis ítems que corresponden a la subescala MA y caracterizan la tendencia hacia el amor posesivo, los celos y la necesidad de asegurar el amor del otro.

Por último, el sexto factor, formado por seis ítems, es coincidente con cinco elementos de LU y uno de AG. Aquí es interesante el análisis del contenido del ítem de AG (Soporto cualquier cosa con tal de estar con una pareja) ya que fue contestado mayoritariamente como "totalmente en desacuerdo" reflejando entonces las características atribuidas a LU. Esto es, el disfrute mutuo sin una intención seria de compromiso.

En función de lo descripto hasta aquí vale la pena considerar si es necesario cambiar la asignación de los ítems para cada estilo de amor dados los resultados del análisis factorial descripto. Si bien los resultados podrían sugerirlo, lo cierto es que la mayoría de los ítems permanecieron agrupados según los tipos supuestos por la teoría de Lee, aunque, tal como ocurrió en el estudio de Hendrick y Hendrick (1986), no se mantiene la distinción entre primarios y secundarios conjeturada por el autor. En los siete ítems que se alinearon en otros tipos que los supuestos, el análisis de su contenido permite pensar que quizá aluden a componentes afines en distintos factores. Estas consideraciones unidas a que el índice de adecuación muestral es correcto pero no el óptimo hacen que no sea prudente introducir cambios respecto de la versión original.

\section{Discusión y Conclusiones}

Al iniciar este estudio nos planteamos como uno de los objetivos estudiar si, en concordancia con los resultados obtenidos en otros países, los hombres 
mostraban una actitud hacia el amor cuyo eje central fuera la diversión y una explícita falta de compromiso en las relaciones amorosas (LUDUS) y las mujeres, una preferencia hacia el amor romántico y pasional (EROS). Los resultados indican que no existen diferencias significativas entre hombres y mujeres en la actitud hacia el amor. Por el contrario, los resultados fueron muy parecidos en ambos sexos y en EROS, AMISTAD, PRAGMA, MANIA y $A G A P E$, el porcentaje de puntajes medios fue el mayoritario en ambos grupos; en tanto que para $L U D U S$, prevalecieron en proporciones similares los puntajes medios y bajos lo cual podría indicar escasa autopercepción del amor en términos sólo de diversión y de escaso compromiso afectivo. Estos resultados contradicen lo encontrado en otros estudios que indican que los varones presentan actitudes sexuales más permisivas e instrumentales en consonancia con un perfil LUDUS en sus estilos del amor (Hendrick, Hendrick, Slapion-Foote \& Foote, 1984).

En relación a esto, un dato interesante es que a diferencia de sus pares norteamericanos (Hendrick y Hendrick, 1986), los hombres entrevistados señalaron en mayor proporción que están enamorados actualmente (aprox. 70\% para varones argentinos y 54\% para los norteamericanos). Quizá sea un indicio que ayude a comprender las diferencias culturales encontradas, en especial la escasa preferencia por Ludus y la tendencia hacia Eros en los varones argentinos.

Esta preferencia por un amor romántico y pasional parece, no obstante, ser algo pródiga si se considera que, respecto de la pregunta acerca de la cantidad de personas con las que habían mantenido relaciones, los hombres argentinos respondieron, en su mayoría, una frecuencia mayor que cinco (aprox. 71\%) y las mujeres, indicaron en mayor proporción la frecuencia de cuatro o menos (aprox. $80 \%$ ). Si bien la diferencia entre varones y mujeres parece importante, no debe olvidarse que estas respuestas pueden estar sujetas a factores de deseabilidad social, necesidades de autopresentación, etc., que pueden distorsionar los resultados ya sea exagerando, ya sea omitiendo.

Otro de los objetivos fue relacionar el tipo de actitud hacia el amor y el estilo de apego en adultos jóvenes. Los resultados obtenidos indican una asociación positiva entre EROS y el tipo de apego seguro, tal como lo sugieren estudios previos (Shaver y Hazan, 1988). No obstante, no se han encontrado asociaciones relevantes, positivas o negativas, entre las restantes dimensiones y tipos de apego.

En cuanto a las propiedades psicométricas de la escala "Actitudes hacia el amor", se ha comprobado que el instrumento revela adecuada consistencia interna y una estructura factorial afín a los supuestos teóricos de Lee, lo que la torna un instrumento adecuado para tareas de investigación aplicada. 


\section{Referencias Bibliográficas}

Ainsworth, M.D.S. (1989). Attachment beyond infancy. American Psychologist, 44, 709-716

Bowlby, J. (1969/1982). Attachment and loss: Vol. 1. Attachment. New York: Basic Books

Hendrick, C. \& Hendrick, S. (1986). A theory and method of love. Journal of Personality and Social Psychology, 50, 2, 392-402

Levy, M.B. \& Davis, K.E. (1988). Love styles and attachment styles compared: Their relations to each other and to various relationship characteristics. Journal of Social and Personal Relationships, 5, 439-471

Shaver, P.R. y Hazan, C. (1988). A biased overview of the study of love. Journal of Social and Personal Relationships, 5, 473-501

Sternberg, R.J. (1986). A triangular theory of love. Psychological Review, 93, 119-135 